\title{
Agegraphic Dark Energy Model in Non-Flat Universe: Statefinder Diagnostic and $w-w^{\prime}$ Analysis.
}

\author{
M. Malekjani* and A. Khodam-Mohammadi ${ }^{\dagger}$ \\ Physics Department, Faculty of Science, \\ Bu-Ali Sina University, Hamedan 65178, Iran
}

\begin{abstract}
We study the interacting agegraphic dark energy (ADE) model in non-flat universe by means of statefinder diagnostic and $w-w^{\prime}$ analysis. First, the evolution of EoS parameter $\left(w_{d}\right)$ and deceleration parameter $(q)$ in terms of scale factor for interacting ADE model in non-flat universe are calculated. Dependence of $w_{d}$ on the ADE model parameters $n$ and $\alpha$ in different spatial curvatures is investigated. We show that the evolution of $q$ is dependent on the type of spatial curvature, beside of dependence on parameters $n$ and $\alpha$. The accelerated expansion takes place sooner in open universe and later in closed universe compare with flat universe. Then, we plot the evolutionary trajectories of the interacting ADE model for different values of the parameters $n$ and $\alpha$ as well as for different contributions of spatial curvature, in the statefinder parameters plane. In addition to statefinder, we also investigate the ADE model in non-flat universe with $w-w^{\prime}$ analysis.
\end{abstract}

\footnotetext{
* E-mail: malekjani@basu.ac.ir

$\dagger$ E-mail: khodam@basu.ac.ir
} 


\section{INTRODUCTION}

In the framework of standard cosmology, the universe is dominated by two mysterious component dark matter and dark energy. WMAP (Wilkinson Microwave Anisotropy Probe) experiment [1], indicates that dark energy occupies about $70 \%$ of the total energy of the universe, and the contribution of dark matter is $\sim 26 \%$. The existence of dark matter is needed to explain the dynamics of galaxies and the formation of structures in the universe [2]. While the dark energy component is responsible for accelerating expansion of the universe. The dark energy (DE) problem is one of the most famous problems in modern cosmology since the discovery of accelerated expansion of the universe. The simplest candidate for dark energy is the cosmological constant in which the equation of state is independent of the cosmic time. This model is the so-called $\Lambda$ CDM, containing a mixture of cosmological constant $\Lambda$ and cold dark matter (CDM). However, two problems arise from this scenario, namely the fine-tuning and the cosmic coincidence problems [3]. In order to solve these two problems, many dynamical dark energy models were suggested, whose equation of state evolves with cosmic time. The models with scale fields such as quintessence [4], phantom field [5], K-essence [6] based on earlier work of $\mathrm{K}$-inflation [7], tachyon field [8], dilaton [9] and quintom [10] suggest that the energy with negative pressure is provided by a scale field evolving down a proper potential. Also the interacting dark energy models including Chaplygin gas [11], holographic dark enrgy models [12], and braneworld models [13] have been proposed. Recently, based on principle of quantum gravity, the agegraphic dark energy (ADE) and the new agegraphic dark energy (NADE) models were proposed by Cai [14] and Wei \& Cai [15], respectively. The ADE model is based on the line of quantum fluctuations of spacetime, the so-called Károlyházy relation $\delta t=\lambda t_{p}^{2 / 3} t^{1 / 3}$, and the energy-time Heisenberg uncertainty relation $E_{\delta t^{3}} \sim t^{-1}$. These relations enable one to obtain an energy density of the metric quantum fluctuations of Minkowski spacetime as follows [16]

$$
\rho_{q} \sim \frac{E_{\delta t^{3}}}{\delta t^{3}} \sim \frac{1}{t_{p}^{2} t^{2}} \sim \frac{m_{p}^{2}}{t^{2}} .
$$

In ADE model the energy density of dark energy is given by Eq.(11). However, in FriedmannRobertson-Walker (FRW) universe, due to effect of curvature, one should assume a numerical factor $3 n^{2}$ in Eq.(11) [14]. The new model of agegraphic dark energy (NADE) has been proposed by Wei and Cai [15], in which the cosmic time is replaced by the conformal time. The 
recent observational data from the Abell Cluster A586 support the interaction between dark matter and dark energy [17]. However, the strength of this interaction is not exactly identified [18]. The ADE and NADE models have been extended regarding the interaction between dark components of the universe, in Refs. [19 22]. Also, the observational experiments such as CMB experiments [23], and the luminosity-distance of supernova measurements [24] reveal the non-flat universe with tiny positive curvature. Hence, we are motivated to consider the non-flat universe containing the interacting dark matter and dark energy components. As was mentioned above, the various dark energy models have been proposed to describe the accelerated phase of the universe. The property of dark energy in these models is strongly model dependent. In order to be capable of differentiating between various models, a sensitive diagnostic tool is needed. The geometrical statefinder diagnostic tool that makes use of parameter pair $\{s, r\}$, introduced by Sahni et al. [25], can discriminate various dark energy models. The statefinder pair is constructed directly from a spacetime metric. The importance of such pair is to distinguish of the cosmological evolution behaviors of dark energy models with the same values of $H_{0}$ and $q_{0}$ at the present time. At future by combining the data of Supernova acceleration probe (SNAP) with statefinder diagnosis, we may choose the best model of dark energy. The statefinder pair $\{s, r\}$ are given by [25]

$$
r=\frac{\dddot{a}}{a H^{3}}, \quad s=\frac{r-1}{3(q-1 / 2)},
$$

Up to now, many authors have studied the dark energy models by means of statefinder diagnostic analysis. The standard $\Lambda$ CDM model and quintessence [25, 26], interacting quintessence models [27, 28], the holographic dark energy models [29, 30], the holographic dark energy model in non-flat universe [31], the phantom model [32], the tachyon [33], the ADE model with and without interaction in flat universe [34] and the interacting NADE model in flat and non-flat universe [35, 36], are analyzed through the statefinder diagnostic. In addition to the statefinder diagnostic, another analysis to discriminate various models of dark energy is $w-w^{\prime}$ analysis which is used widely in the literature [34, 37].

In this paper we study the ADE model in a non-flat universe. First, the evolutionary behavior of EoS parameter, $w_{d}$, and deceleration parameter $q$ for different illustrative values of model parameters $n$ and $\alpha$ ( $\alpha$ is the interaction parameter between dark matter and dark energy) in non-flat universe are investigated. Then we examine the ADE model in a non-flat universe by means of statefinder diagnostic tool and $w-w^{\prime}$ analysis. 


\section{THE ADE MODEL IN NON-FLAT UNIVERSE}

As we mentioned in Sec. (II), the energy density of dark energy in ADE model is given by

$$
\rho_{d}=\frac{3 n^{2} m_{p}^{2}}{T^{2}}
$$

where the cosmic time $T$ is defined as the age of the universe

$$
T=\int_{0}^{t} d t=\int_{0}^{a} \frac{d a}{H a} .
$$

Assuming a non-flat Friedman-Robertson-Walker (FRW) universe containing the agegraphic dark energy and cold dark matter components, the corresponding freidmann equation is as follows

$$
H^{2}+\frac{k}{a^{2}}=\frac{1}{3 m_{p}^{2}}\left(\rho_{m}+\rho_{d}\right)
$$

where $k=1,0,-1$ is curvature parameter corresponding to a closed, flat and open universe, respectively. Recent observations support a closed universe with a tiny positive small curvature $\Omega_{k 0}=1 / H_{0}^{2} \simeq 0.02[1]$. The other form of Friedmann equation with respect to fractional energy density $\Omega_{i}=\rho_{i} / \rho_{c}$, and critical density $\rho_{c}=3 m_{p}^{2} H^{2}$ is

$$
\Omega_{m}+\Omega_{d}=1+\Omega_{k}
$$

From Eq.(3), it is easy to find that

$$
\Omega_{d}=\frac{n^{2}}{H^{2} T^{2}}
$$

The continuity equations for interacting dark energy and dark matter are given by

$$
\begin{aligned}
\dot{\rho_{m}}+3 H \rho_{m} & =Q, \\
\dot{\rho}_{d}+3 H\left(\rho_{d}+p_{d}\right) & =-Q,
\end{aligned}
$$

Three forms of $Q$ which have been extensively used in the literatures [15, 35? ] are

$$
Q=9 \alpha_{i} m_{p}^{2} H^{3} \Omega_{i} ; \quad \Omega_{i}= \begin{cases}\Omega_{d} ; & i=1 \\ \Omega_{m} ; & i=2 \\ \Omega_{d}+\Omega_{m} ; & i=3\end{cases}
$$


Differentiating Eq.(17) and using Eqs.(3), (15), (6) and (9), the derivative of $\Omega_{d}$ can be calculated as

$$
\begin{aligned}
& \Omega_{d}^{\prime}=\frac{\dot{\Omega}}{H}=-2 \Omega_{d}\left[\frac{\dot{H}}{H^{2}}+\frac{\sqrt{\Omega_{d}}}{n}\right] \\
& \frac{\dot{H}}{H^{2}}=-\frac{\Omega_{d}^{3 / 2}}{n}-\frac{3}{2}\left(1-\Omega_{d}\right)-\frac{\Omega_{k}}{2}+\frac{Q_{c}}{2},
\end{aligned}
$$

where prime denotes the derivative with respect to $\ln a$ and $Q_{c}=Q / H \rho_{c}=3 \alpha_{i} \Omega_{i}$. The relations (11) and (12), also has been obtained in [22] for third interaction form of $Q$.

Substituting the relation (12) in Eq.(11), we obtain a normal differential equation for $\Omega_{d}$ as

$$
\Omega_{d}^{\prime}=\Omega_{d}\left[\left(1-\Omega_{d}\right)\left(3-2 \frac{\sqrt{\Omega_{d}}}{n}\right)+\Omega_{k}-Q_{c}\right]
$$

where $\Omega_{k}$ is given by

$$
\Omega_{k}=\frac{a \gamma\left(1-\Omega_{d}\right)}{1-a \gamma}
$$

Here $\gamma$ satisfies the following equation

$$
\frac{\Omega_{k}}{\Omega_{m}}=a \frac{\Omega_{k 0}}{\Omega_{m 0}}=a \gamma
$$

From the continuity equation (i.e., Eq9) and Eqs.(3).7), it is easy to find that the EoS parameter of the interacting agegraphic dark energy, $w_{d}=p_{d} / \rho_{d}$, can be obtained as

$$
w_{d}=-1-Q_{c d}+\frac{2 \sqrt{\Omega_{d}}}{3 n}
$$

where $Q_{c d}=Q_{c} /\left(3 \Omega_{d}\right)$. Using the Eq.(13), the evolutionary behavior of $w_{d}$ is given by the following equation

$$
w_{d}^{\prime}=+\frac{\sqrt{\Omega_{d}}}{3 n}\left[1+\Omega_{k}-3 \Omega_{d}-2 \frac{\sqrt{\Omega_{d}}}{n}\left(1-\Omega_{d}\right)-Q_{c}\right]-Q_{c d}^{\prime} .
$$

The deceleration parameter $q$ can be obtained as

$$
\begin{aligned}
q & =-\frac{\dot{H}}{H^{2}}-1=\frac{\left(1+\Omega_{k}\right)}{2}+\frac{3}{2} \Omega_{d} w_{d} \\
& =\frac{\Omega_{d}^{3 / 2}}{n}-\Omega_{d}+\frac{1-\Omega_{d}}{2(1-a \gamma)}-\frac{Q_{c}}{2}
\end{aligned}
$$




\section{STATEFINDER DIAGNOSTIC FOR INTERACTING ADE MODEL IN NON- FLAT UNIVERSE}

Now we switch to the statefinder pair $\{\mathrm{r}, \mathrm{s}\}$, which was expressed in Sec \. From the definition of $q$ and $H$, the parameter $r$ in Eq.(2) can be written as

$$
r=\frac{\ddot{H}}{H^{3}}-3 q-2 \text {. }
$$

Using Eqs.(12), (16) and (18), we have

$$
\frac{\ddot{H}}{H^{3}}=\frac{9}{2}+\frac{9}{2} \Omega_{d} w_{d}\left(w_{d}+Q_{c d}+2\right)-\frac{3}{2} \Omega_{d} w_{d}^{\prime}+\frac{5}{2} \Omega_{k} .
$$

Hence, the Eq.(20) can be obtained as

$$
r=1+\Omega_{k}+\frac{9}{2} \Omega_{d} w_{d}\left(w_{d}+Q_{c d}+1\right)-\frac{3}{2} \Omega_{d} w_{d}^{\prime}
$$

In a non flat universe, Evans et al. [38] generalize the definition of parameter $s$ in Eq.(2) as

$$
s=\frac{r-\Omega_{t o t}}{\frac{3}{2}\left(q-\frac{\Omega_{t o t}}{2}\right)},
$$

where the total fractional energy density is $\Omega_{t o t}=\Omega_{m}+\Omega_{d}=1+\Omega_{k}$. Therefore from this new definition we have

$$
s=1+w_{d}+Q_{c d}-\frac{w_{d}^{\prime}}{3 w_{d}} .
$$

By omitting $w_{d}^{\prime}$ between (22) and (24), we find $r$ in terms of $s$ as follows

$$
r=1+\Omega_{k}+\frac{9}{2} s \Omega_{d} w_{d}
$$

\section{NUMERICAL RESULTS}

In this section, first we calculate the EoS parameter, $w_{d}$, of ADE model and deceleration parameter, $q$, for different model parameters $n$ and $\alpha$ in non flat universe. Then we study the ADE model in non flat universe by means of statefinder diagnostic tool and $w-w^{\prime}$ analysis. Here we assume the first case of interaction form, $Q=Q_{1}=9 \alpha m^{2} H^{3} \Omega_{d}$, in Eq.(10). In this case, $Q_{c}=3 \alpha \Omega_{d}, Q_{c d}=\alpha$ and the differential equation for $\Omega_{d}$ (i.e., Eq.13) can be reduced as

$$
\Omega_{d}^{\prime}=\Omega_{d}\left[\left(1-\Omega_{d}\right)\left(3-\frac{2 \sqrt{\Omega_{d}}}{n}\right)-3 \Omega_{d} \alpha+\Omega_{k}\right]
$$


The Eqs.(16), (17), (22) and (24), reduced as follows

$$
\begin{gathered}
w_{d}=-1+\frac{2 \sqrt{\Omega_{d}}}{3 n}-\alpha \\
w_{d}^{\prime}=\frac{\sqrt{\Omega_{d}}}{3 n}\left[\left(1+\Omega_{k}\right)-3 \Omega_{d}(1+\alpha)-\frac{2 \sqrt{\Omega_{d}}}{n}\left(1-\Omega_{d}\right)\right] \\
r=1+\Omega_{k}+\frac{9}{2} \Omega_{d} w_{d}\left(w_{d}+\alpha+1\right)-\frac{3}{2} \Omega_{d} w_{d}^{\prime} . \\
s=1+w_{d}+\alpha-\frac{w_{d}^{\prime}}{3 w_{d}} .
\end{gathered}
$$

From Eq.(27), it is easy to see that in the absence of interaction $(\alpha=0.0) w_{d}$ is larger than -1 , and the ADE model can not cross the phantom divide. In the presence of interaction between dark energy and dark matter the ADE model can cross the phantom divide, if $\alpha>2 \sqrt{\Omega_{d}} / 3 n$. Taking $\Omega_{d}=0.72$ for the present time, the phantom-like EoS can be obtained if $n \alpha>0.565$. The WMAP and SDSS observational experiments indicate that the best value for $n$ is 3.4 [39]. Thus, the condition $w_{d}<-1$ leads to $\alpha>0.166$. For example for $\alpha=0.2$ we get $w_{d}=-1.03$ at the present time. Hence, the phantom-like equation of state can be generated from an interacting ADE model in the universe with any spacial curvature.

Fig.(1) shows the evolution of $w_{d}$ in terms of scale factor, $a$, for different model parameters $n$ and $\alpha$ and various spatial curvatures. In first arrow panels, $w_{d}$ is plotted in the absence of interaction term between dark matter and dark energy $(\alpha=0.0)$. In this case the phantom divide can not be crossed for any spatial curvature. In second and third arrow panels, the interaction between dark matter and dark energy is taking into account. For $\alpha=0.1$, the phantom divide is achieved for various spatial curvature. Increasing the parameter $n$ leads to smaller value of $w_{d}$. In third arrow, $\alpha=0.2$, the treatment of $w_{d}$ may be different form previous cases. In the case of $(n=0.9, \alpha=0.2), w_{d}$ is positive at the early time, $w_{d}>0$. This behavior of $w_{d}$ at the early time is due to the presence of $2 \sqrt{\Omega_{d}} / 3 n$ in Eq.(27). This term leads to larger value of $w_{d}$ for smaller value of $n$. It should be noted that for smaller value of $n$ and larger value of $\alpha$, we get a larger value of $\Omega_{d}$ at the early time (see Fig.(1) of Ref.[20]). Hence, it is easy to see that $w_{d}$ tends to the larger values because of the presence of $n$ at denominator and $\Omega_{d}$ at numerator of Eq.(27). For instance for the model parameters $n=0.9, \alpha=0.2$, at the scale factor $a=0.01$, $2 \sqrt{\Omega_{d}} / 3 n=1.3$ which is bigger than $1+\alpha=1.2$, thus $w_{d}>0$. For the model parameters 
$n=1.2, \alpha=0.2$, the ADE model can cross the phantom divide in the flat and closed universe, but in open universe it can not cross the phantom divide. In the case of ( $n=2$, $\alpha=0.2)$ the phantom divide can be achieved for any spacial curvature. By comparing with NADE model which has been investigated by us [36], we see that in ADE model, for a given value of $\alpha$, the phantom divide is achieved for smaller $n$ at early time. For example, for $\alpha=0.1$, the ADE model with $n=0.9$ can cross the phantom divide at the early time (see Fig.1), while in NADE model it has been achieved, if $n>3$ (see Fig.1-b of Ref.[36]).

The other cosmological parameter which we calculate it, is the deceleration parameter $q$. The parameter $q$ in ADE model is given by Eq.(18). In the early time, where $\Omega_{d} \rightarrow 0$ and $\Omega_{k} \rightarrow 0$, the parameter $q$ converges to $1 / 2$, whereas the universe has been dominated by dark matter. In Fig(2), we show the evolution of $q$ as a function of cosmic scale factor for different model parameters $n$ and $\alpha$ and also various contribution of spatial curvature of the universe. In first arrow panels, the ADE model without interaction term $(\alpha=0.0)$, we see that increasing the parameter $n$ leads to smaller value for $q$. The transition from decelerated expansion $(q>0)$ to accelerated expansion $(q<0)$ tacks place sooner for larger value of $n$. For the parameters $n=0.9, \alpha=0.0$, the accelerated universe can not be archived even at the late time. The transition from decelerated to the accelerated universe occurs gradually from open, flat and closed universe. However, the difference between them is very small, but we can interpret that the accelerated phase occurs earlier in open universe. In second arrow panels, we consider $\alpha=0.1$. Here, in this case, the universe enters into the accelerated phase earlier compare with the pervious case $(\alpha=0.0)$. In third arrow panels, the interaction parameter is assumed as $\alpha=0.2$. We see that in this case the accelerated phase is achieved sooner than previous cases. Hence, the universe enters the accelerated phase earlier by increasing the interaction parameter $\alpha$.

At following, we calculate the evolutionary trajectories in the statefinder plane and analyze the interacting ADE model in non-flat universe with statefinder point of view. The statefinder is a geometrical diagnostic tool, because it depends only on the scale factor $a$. The standard $\Lambda \mathrm{CDM}$ model corresponds to a fixed point $\{\mathrm{r}=1, \mathrm{~s}=0\}$ in the $\mathrm{r}$-s diagram in a flat universe [25], and $\left\{r=1+\Omega_{k}, s=0\right\}$ in a non-flat universe [38]. It should be mentioned that the statefinder diagnostic for ADE model in flat universe has been investigated in 
Ref.[34], where the focus is put on the diagnostic of the different values of parameters $n$ and $\alpha$. In Ref.[34], it has been discussed that from the statefinder viewpoint $n$ and $\alpha$ play the significant role in this model and it leads to the values of $\{r, s\}$ in today and future tremendously different. Here we want to focus on the statefinder diagnostic of the spatial curvature contribution in the ADE model.

The Eqs.(29]30) and (28) describe the evolution of statefinder parameters $\{r, s\}$ and also $w_{d}^{\prime}$. Since $\Omega_{d} \rightarrow 0$ at the early time, thus from Eq.(28) one can see that $w_{d}^{\prime} \rightarrow 0$, at this time. It is worth noting that $\Omega_{k}$ tends to zero at the early time. Using Eqs. (29)30), we see that the ADE model in the early time, independent of model parameters $n$ and $\alpha$ and any contribution of spatial curvature, gives the fixed point $(r=1, s=0)$ in $r-s$ diagram.

In Fig.(3) the evolutionary trajectories of statefinder for the interacting ADE model is plotted. While the universe expands, the trajectories of the statefinder start from the fixed point $\{\mathrm{r}=1, \mathrm{~s}=0\}$ at the early time and then the parameter $s$ increases and the parameter $r$ decreases. In first arrow panels, the ADE is considered without interaction term $(\alpha=0.0)$. We see that, in addition to the model parameters $n$ and $\alpha$, the evolutionary trajectory is dependent on the model curvature of the universe. The colore points on the curves represent the today's values of statefinder parameters $\left(r_{0}, s_{0}\right)$. The present value $r_{0}$ in closed universe is largest, while $s_{0}$ is smallest compare with flat and open universe. Also, larger value of $n$ obtains the larger $r_{0}$ and smaller $s_{0}$. In second arrow panels, the interaction parameter is chosen as $\alpha=0.1$. In this case, we see that the present values $\left(r_{0}, s_{0}\right)$ are smaller compare with the case of $\alpha=0.0$. In third arrow panels the interaction parameter is $\alpha=0.2$. The present values $\left(r_{0}, s_{0}\right)$ is smallest compare with previous cases of $\alpha$. For the parameteres $(n=1.2, \alpha=0.2)$, the evolutionary trajectory in open universe has a different treatment in comparison with of flat and closed universe. The today's values $\left(r_{0}, s_{0}\right)$ for different contribution of spatial curvature and model parameters $n$ and $\alpha$ are collected in table(1).

Finally, we do the $w-w^{\prime}$ analysis for interacting ADE model in non flat universe. In this analysis, the standard $\Lambda$ CDM model corresponds to the fixed point $\left\{w_{d}=-1, w_{d}^{\prime}=0\right\}$ in the $w-w^{\prime}$ plane. The evolution of $w_{d}$ and $w_{d}^{\prime}$ is given by Eqs.(27, 28). In Fig.(4) the evolutionary trajectories in $w-w^{\prime}$ plane are shown for different model parameters $n$ and $\alpha$ and also the contribution of different spatial curvatures. The first arrow panels, show the evolutionary trajectories for ADE model without interaction term $(\alpha=0.0)$. In this case, 
from Eqs.(27, 28) it is easy to see that at the early time $w_{d}=-1.0$ and $w_{d}^{\prime}=0.0$. By expanding the universe, the trajectories start from the point $\left(w=-1, w^{\prime}=0.0\right)$, then $w_{d}$ increases and also $w_{d}^{\prime}$ increases to a some maximum value, after that decreases in $w-w^{\prime}$ plane. At the early time, the contribution of spatial curvature can be neglected, and the evolutionary trajectories start from the fixed point $\left(w=-1, w^{\prime}=0.0\right)$ for any spatial curvature. The colore points on the curves represent the present values of $\left\{w_{d}, w^{\prime}\right\}$. One can see that different spatial curvatures of the universe result different evolutionary trajectories in $w-w^{\prime}$ plane. The present $w_{d}$ is equal for all spatial curvature models, but $w_{d}^{\prime}$ in closed universe is largest compare with open and flat universe. The evolutionary trajectory is also dependent on the model parameters $n$ and $\alpha$. Different values of $n$ result the different trajectories. The maximum of $w_{d}^{\prime}$ is smaller when $n$ is larger. Also, the present value of $w_{d}$ is smaller and the present value of $w_{d}^{\prime}$ is larger when the parameter $n$ is larger. In second arrow panels, the trajectories are plotted for $\alpha=0.1$. In this case, from Eqs.(27, 28), it is obvious that the initial values of $w_{d}$ and $w_{d}^{\prime}$ at the early time are $w_{d}=-1.1$ and $w_{d}^{\prime}=0.0$. In this case the present $w_{d}$ and $w_{d}^{\prime}$ is smaller compare with the previous case $(\alpha=0.0)$. In third arrow panels, the evolutionary trajectories are shown for $\alpha=0.2$. For the parameters ( $n=0.9, \alpha=0.2$ ), we see the different behavior of evolutionary trajectories. The trajectories start form the point $w_{d}=0.1, w_{d}^{\prime}=0.0$, then $w_{d}$ decreases, and $w_{d}^{\prime}$ decreases to a minimum value, after that increases. In the case of $n=1.2, \alpha=0.2$, the trajectories in open universe is completely different from flat and closed universe. Similar with the previous cases, in the case of $\alpha=0.2$, the present values of $w_{d}, w_{d}^{\prime}$ are larger when $n$ is larger. The present values of $w_{d}, w_{d}^{\prime}$ for different model parameters $n$ and $\alpha$ and spatial curvature $\Omega_{k}$, are presented in table (2).

\section{CONCLUSION}

In this paper, the interacting ADE model has been extended in a non flat universe. This extension can be summarized as:

( $i$ ) we studied the evolutionary treatment of EoS parameter, $w_{d}$, and the deceleration parameter, $q$, in different contribution of spatial curvature. We showed that for any spatial curvature, the ADE model without interaction term can not cross the phantom divide. To achieve the phantom divide at any contribution of curvature, the interaction term is needed. 
The ADE model gives the decelerated expansion at the early time and then accelerated expansion later. The transition from decelerated phase to accelerated phase is dependent on parameters $n$ and $\alpha$ as well as the type of spatial curvature of the universe. The universe undergoes the accelerated expansion earlier, for larger values of $n$ and $\alpha$. Also for the same values of $n$ and $\alpha$, the accelerated phase in open universe tacks place sooner compare with flat and closed universe.

(ii) We performed the statefinder diagnostic and $w-w^{\prime}$ analysis for the interacting ADE model in non-flat universe. The statefinder diagnostic tool and $w-w^{\prime}$ analysis are useful methods to discriminate the various models of dark energy. Moreover, the present values of $\{\mathrm{r}, \mathrm{s}\}$ and $\left\{w, w^{\prime}\right\}$, if can be extracted from precise observational data in a model-independent way, can be as a possible discriminator for testing the cosmological models of dark energy. We showed that the evolutionary trajectories in statefinder parameters plane and $w-w^{\prime}$ plane are different for various types of spatial curvatures. Also, the present values of $\{\mathrm{r}, \mathrm{s}\}$ and $\left\{w, w^{\prime}\right\}$ are different for various spatial curvatures. Hence, the statefinder diagram and $w-w^{\prime}$ analysis showed that the contributions of the spatial curvature in the model can be diagnosed out explicitly in this methods. We hope that the future high-precision observations such as the SNAP-type experiment be capable to determine the statefinder parameters precisely and consequently single out the right cosmological models.

[1] C. L. Bennett et al., Astrophys.J.Suppl. 148, 1 (2003);

D. N. Spergel et al., Astrophys.J.Suppl. 148, 175 (2003);

D.N.Spergel et al., Astrophys.J.Suppl. 170, 377 (2007).

[2] Bosma, A. 1981, AJ, 86, 1825;

Peebles, P. J. E., 1980. The Large-Scale Structure of the Universe. Princeton University Press, NJ.

[3] E. J. Copeland, M. Sami, S. Tsujikawa, Int. J. Mod. Phys. D 15, 1753(2006).

[4] C. Wetterich, Nucl. Phys. B 302, 668 (1988);

B. Ratra, J. Peebles, Phys. Rev. D 37, 321 (1988). 
[5] R. R. Caldwell, Phys. Lett. B 545, 23 (2002);

S. Nojiri, S.D. Odintsov, Phys. Lett. B 562, 147 (2003);

S. Nojiri, S.D. Odintsov, Phys. Lett. B 565, 1 (2003).

[6] T. Chiba, T. Okabe, M. Yamaguchi, Phys. Rev. D 62, 023511(2000);

C. Armendariz-Picon, V. Mukhanov, P.J. Steinhardt, Phys. Rev. Lett. 85, 4438 (2000);

C. Armendariz-Picon, V. Mukhanov, P.J. Steinhardt, Phys. Rev. D 63, 103510 (2001).

[7] C. Armendariz-Picon, T. Damour, V. Mukhanov, Phys. Lett. B 458, 209 (1999);

J. Garriga, V. Mukhanov, Phys. Lett. B 458, 219 (1999).

[8] A. Sen, J. High Energy Phys. 04, 048 (2002);

T. Padmanabhan, Phys. Rev. D 66, 021301 (2002);

T. Padmanabhan, T.R. Choudhury, Phys. Rev. D 66, 081301 (2002).

[9] M. Gasperini, F. Piazza, G. Veneziano, Phys. Rev. D 65, 023508 (2002);

N. Arkani-Hamed, P. Creminelli, S. Mukohyama, M. Zaldarriaga, JCAP. 04, 001 (2004);

F. Piazza, S. Tsujikawa, J. Cosmol. Astropart. Phys. 07, 004 (2004).

[10] E. Elizalde, S. Nojiri, S.D. Odinstov, Phys. Rev. D 70, 043539 (2004);

S. Nojiri, S.D. Odintsov, S. Tsujikawa, Phys. Rev. D 71, 063004 (2005);

A. Anisimov, E. Babichev, A. Vikman, J. Cosmol. Astropart. Phys. 06, 006 (2005).

[11] A. Kamenshchik, U. Moschella, V. Pasquier, Phys. Lett. B 511, 265 (2001);

M.C. Bento, O. Bertolami, A.A. Sen, Phys. Rev. D 66, 043507 (2002).

[12] A. Cohen, D. Kaplan, A. Nelson, Phys. Rev. Lett. 82, 4971 (1999);

P. Horava, D.Minic, Phys. Rev. Lett. 85, 1610 (2000);

S. Thomas, Phys. Rev. Lett. 89, 081301 (2002);

M. Li, Phys. Lett. B 603, 1 (2004);

A. Sheykhi, Phys. Lett. B 681, 205 (2009);

P. Horava, D. Minic, Phys. Rev. Lett. 509 (2001) 138;

K. Enqvist, M. S. Sloth, Phys. Rev. Lett. 93 (2004) 221302.

S. D. H. Hsu, Phys. Lett. B 594 (2004) 13.

M. R. Setare, S. Shafei, JCAP 09 (2006) 011;

M. R. Setare, Phys. Lett. B 644 (2007) 99;

M. R. Setare, E. C. Vagenas, Phys. Lett. B 666 (2008) 111;

M. R. Setare, Phys. Lett. B 642 (2006) 421; 
H. M. Sadjadi, arXiv:0902.2462.

B. Wang, C. Y. Lin and E. Abdalla, Phys. Lett. B 637 (2005) 357;

M. R. Setare, Eur. Phys. J. C 52 (2007) 689;

Bin Wang, et al., astro-ph/0607126.

[13] C. Deffayet, G.R. Dvali, G. Gabadadze, Phys. Rev. D 65, 044023 (2002);

V. Sahni, Y. Shtanov, J. Cosmol. Astropart. Phys. 11, 014 (2003).

[14] R. G. Cai, Phys. Lett. B 657 (2007) 228.

[15] H. Wei \& R. G. Cai, Phys. Lett. B 660, 113 (2008).

[16] M. Maziashvili Int. J. Mod. Phys. D 16 (2007) 1531;

M. Maziashvili, Phys. Lett. B 652 (2007) 165.

[17] O. Bertolami, F. Gil Pedro, Le Delliou, M., Phys. Lett. B 654, 165 (2007).

[18] C. Feng, B. Wang, Y. Gong, R. K. Su, J. Cosmol. Astropart. Phys. 0709, 005 (2007).

[19] H. Wei, R.G. Cai, Phys. Lett. B 663, 1 (2008).

[20] H. Wei, R.G. Cai, Eur. Phys. J. C 59, 99 (2009).

[21] Y.W. Kim et al., Mod. Phys. Lett. A 23, 3049 (2008);

K.Y. Kim, H.W. Lee, Y.S. Myung, Phys. Lett. B 660, 118 (2008);

J.P. Wu, D.Z. Ma, Y. Ling, Phys. Lett. B 663, 152 (2008);

J. Zhang, X. Zhang, H. Liu, Eur. Phys. J. C 54, 303 (2008);

I.P. Neupane, Phys. Lett. B 673, 111 (2009).

[22] A. Sheykhi, Phys. Lett. B 680, 113 (2009).

[23] J. L. Sievers, et al., Astrophys. J. 591, 599 (2003);

C.B. Netterfield, et al., Astrophys. J. 571, 604 (2002);

A. Benoit, et al., Astron. Astrophys. 399 (2003) L25;

A. Benoit, et al., Astron. Astrophys.399 (2003) L19.

[24] R. R. Caldwell, M. Kamionkowski, astro-ph/0403003;

B. Wang, Y. G. Gong, R. K. Su, Phys. Lett. B 605 (2005) 9.

[25] V. Sahni, T. D. Saini, A. A. Starobinsky and U. Alam, JETP Lett. 77, 201 (2003).

U. Alam, V. Sahni, T. D. Saini and A. A. Starobinsky, MNRAS. 344, 1057 (2003)

[26] U. Alam, V. Sahni, T. D. Saini and A. A. Starobinsky, Mon. Not. Roy. Astron. Soc. 344, 1057 (2003) astro-ph/0303009.

[27] W. Zimdahl and D. Pavon, Gen. Rel. Grav. 36, 1483 (2004) gr-qc/0311067. 
[28] X. Zhang, Phys. Lett. B 611, 1 (2005) astro-ph/0503075].

[29] X. Zhang, Int. J. Mod. Phys. D 14, 1597 (2005) astro-ph/0504586].

[30] J. Zhang, X. Zhang and H. Liu, arXiv:0705.4145 [astro-ph].

[31] M. R. Setare, J. Zhang and X. Zhang, JCAP 0703, 007 (2007) (gr-qc/0611084].

[32] B. R. Chang, H. Y. Liu, L. X. Xu, C. W. Zhang and Y. L. Ping, JCAP 0701, 016 (2007) astro-ph/0612616.

[33] Y. Shao and Y. Gui, gr-qc/0703111.

[34] H. Wei, R. G. Cai, Phys. Lett. B 655, 1(2007).

[35] L. Zhang, J. Cui, J. Zhang \& X. Zhang, Int. J. Mod. Phys. D 19, 21 (2010).

[36] A. Khodam-Mohammadi, M. Malekjani, (2010), [gr-qc/1003.0543].

[37] R. J. Scherrer, Phys. Rev. D 73, 043502 (2006) astro-ph/0509890].

T. Chiba, Phys. Rev. D 73, 063501 (2006) astro-ph/0510598.

V. Barger, E. Guarnaccia and D. Marfatia, Phys. Lett. B 635, 61 (2006) hep-ph/0512320.

W. Zhao, Phys. Rev. D 73, 123509 (2006) astro-ph/0604460];

W. Zhao, arXiv:0706.2211 [astro-ph].

G. Calcagni and A. R. Liddle, Phys. Rev. D 74, 043528 (2006) astro-ph/0606003.

Z. K. Guo, Y. S. Piao, X. M. Zhang and Y. Z. Zhang, Phys. Rev. D 74, 127304 (2006) astro-ph/0608165.

Z. G. Huang, H. Q. Lu and W. Fang, hep-th/0610018;

Z. G. Huang, X. H. Li and Q. Q. Sun, Astrophys. Space Sci. 310, 53 (2007) hep-th/0610019].

R. de Putter and E. V. Linder, arXiv:0705.0400 [astro-ph].

[38] A. K. D. Evans, I. K. Wehus, O. Gron and O. Elgaroy, Astron. Astrophys. 430, 399 (2005).

[39] L. Amendola, Phys. Rev. D 60 (1999) 043501;

L. Amendola, Phys. Rev.D 62 (2000) 043511;

L. Amendola and C. Quercellini, Phys. Rev. D 68 (2003) 023514;

L. Amendola and D. T. Valentini, Phys. Rev. D 66 (2002) 043528. 
TABLE I: The present values of the statefinder parameters $(r, s)$ for different model parameters $n$ and $\alpha$ in closed, flat and open universe.

\begin{tabular}{crrr}
\hline$\Omega_{k 0}=0.02$ (Closed universe) & $n=0.9$ & $n=1.2$ & $n=2.0$ \\
\hline$\alpha=0.0$ & $(0.162,0.730)$ & $(0.102,0.545)$ & $(0.2770,0.3261)$ \\
$\alpha=0.1$ & $(0.029,0.660)$ & $(0.003,0.506)$ & $(0.217,0.308)$ \\
$\alpha=0.2$ & $(-0.102,0.614)$ & $(-0.095,0.478)$ & $(0.158,0.294)$ \\
\hline$\Omega_{k 0}=0.0$ (Flat universe) & $n=0.9$ & $n=1.2$ & $n=2.0$ \\
\hline$\alpha=0.0$ & $(0.133,0.718)$ & $(0.070,0.538)$ & $(0.240,0.323)$ \\
$\alpha=0.1$ & $(0.002,0.650)$ & $(-0.028,0.500)$ & $(0.181,0.305)$ \\
$\alpha=0.2$ & $(-0.130,0.606)$ & $(-0.127,0.473)$ & $(0.121,0.292)$ \\
\hline$\Omega_{k 0}=-0.02$ (Open universe) & $n=0.9$ & $n=1.2$ & $n=2.0$ \\
\hline$\alpha=0.0$ & $(0.109,0.707)$ & $(0.042,0.533)$ & $(0.2091,0.3208)$ \\
$\alpha=0.1$ & $(-0.022,0.642)$ & $(-0.056,0.496)$ & $(0.149,0.303)$ \\
$\alpha=0.2$ & $(-0.154,0.599)$ & $(-0.155,0.469)$ & $(0.090,0.290)$ \\
\hline
\end{tabular}


TABLE II: The present values of the parameters $\left(w, w^{\prime}\right)$ for different model parameters $n$ and $\alpha$ in closed, flat and open universe.

\begin{tabular}{crrr}
\hline$\Omega_{k 0}=0.02$ (Closed universe) & $n=0.9$ & $n=1.2$ & $n=2.0$ \\
\hline$\alpha=0.0$ & $(-0.367,0.107)$ & $(-0.525,0.111)$ & $(-0.7152,0.088)$ \\
$\alpha=0.1$ & $(-0.467,0.038)$ & $(-0.625,0.059)$ & $(-0.815,0.057)$ \\
$\alpha=0.2$ & $(-0.567,-0.030)$ & $(-0.725,0.007)$ & $(-0.915,0.026)$ \\
\hline$\Omega_{k 0}=0.0$ (Flat universe) & $n=0.9$ & $n=1.2$ & $n=2.0$ \\
\hline$\alpha=0.0$ & $(-0.367,0.094)$ & $(-0.525,0.101)$ & $(-0.715,0.082)$ \\
$\alpha=0.1$ & $(-0.467,0.024)$ & $(-0.625,0.049)$ & $(-0.815,0.051)$ \\
$\alpha=0.2$ & $(-0.567,-0.044)$ & $(-0.725,-0.003)$ & $(-0.915,0.020)$ \\
\hline$\Omega_{k 0}=-0.02$ (Open universe) & $n=0.9$ & $n=1.2$ & $n=2.0$ \\
\hline$\alpha=0.0$ & $(-0.367,0.082)$ & $(-0.525,0.092)$ & $(-0.715,0.077)$ \\
$\alpha=0.1$ & $(-0.467,0.013)$ & $(-0.625,0.040)$ & $(-0.815,0.046)$ \\
$\alpha=0.2$ & $(-0.567,-0.056)$ & $(-0.725,-0.011)$ & $(-0.915,0.014)$ \\
\hline
\end{tabular}



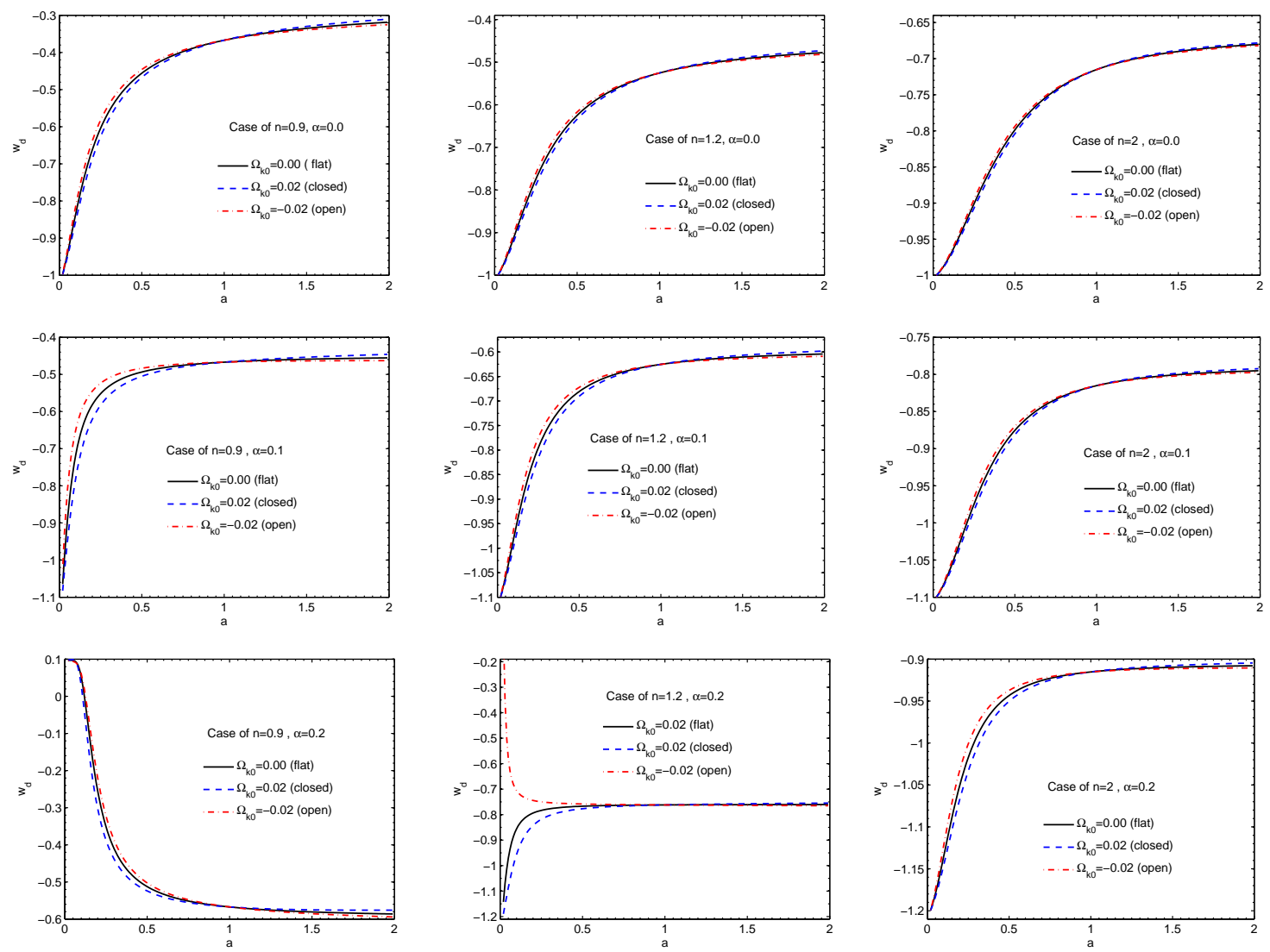

FIG. 1: The evolution of EoS parameter, $w_{d}$, versus of $a$ for different model parameters $n$ and $\alpha$ in different closed, flat and open universe. In first arrow, in the absence of interaction between dark matter and dark energy, the phantom divide can not be achieved. In second and third arrows, in the presence of interaction, the phantom divide can be achieved. 

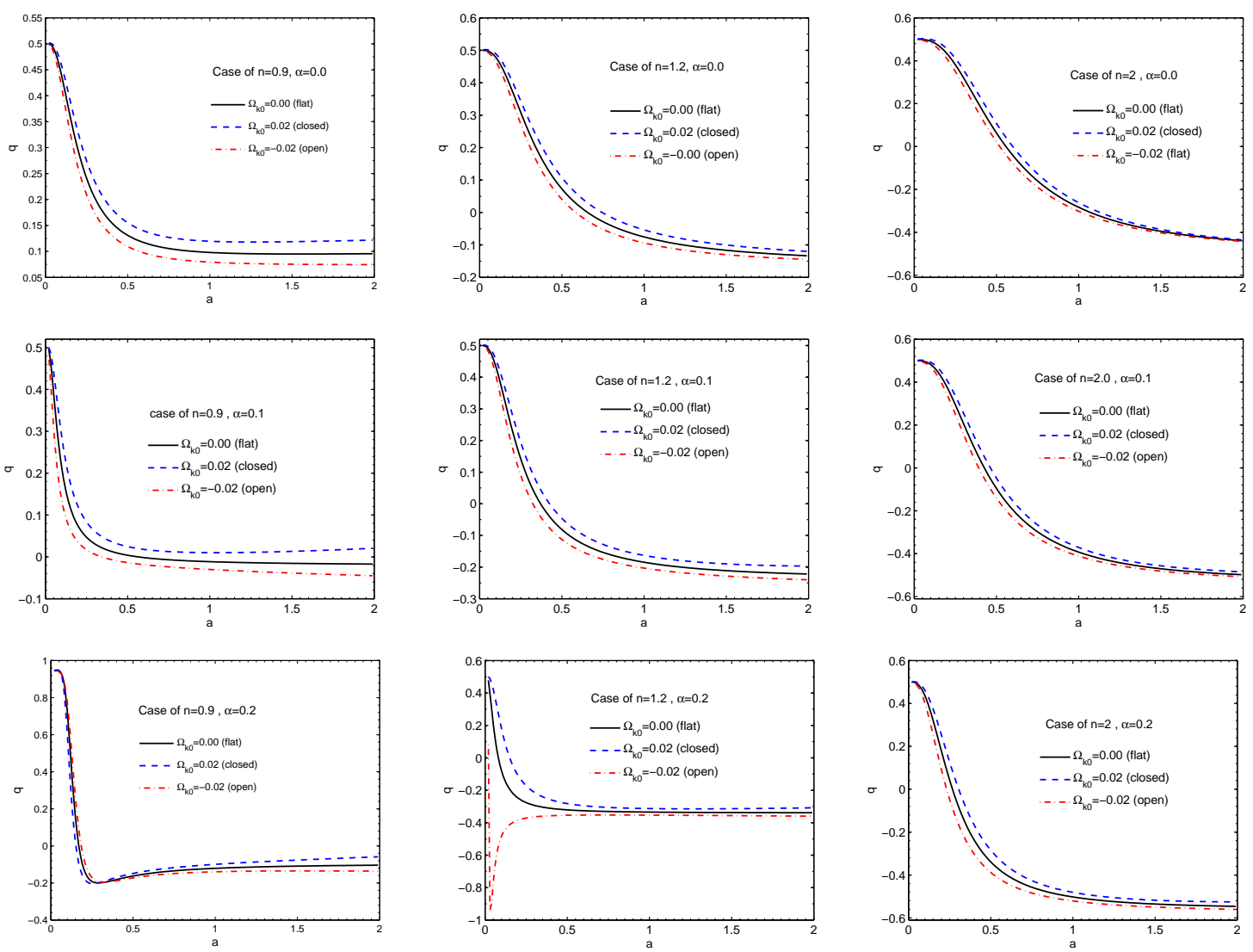

FIG. 2: The evolution of deceleration parameter, $q$, versus of $a$ for different model parameters $n$ and $\alpha$ in different closed, open flat and open universe. In first arrow the ADE model is assumed without interaction term between dark components of the universe, while in the second and third arrows the interaction term is included. The universe undergoes accelerated expansion earlier, for larger values of $n$ and $\alpha$. Also, for the same values of parameters $n$ and $\alpha$ the transition from decelerated to the accelerated expansion occurs sooner in open universe. 

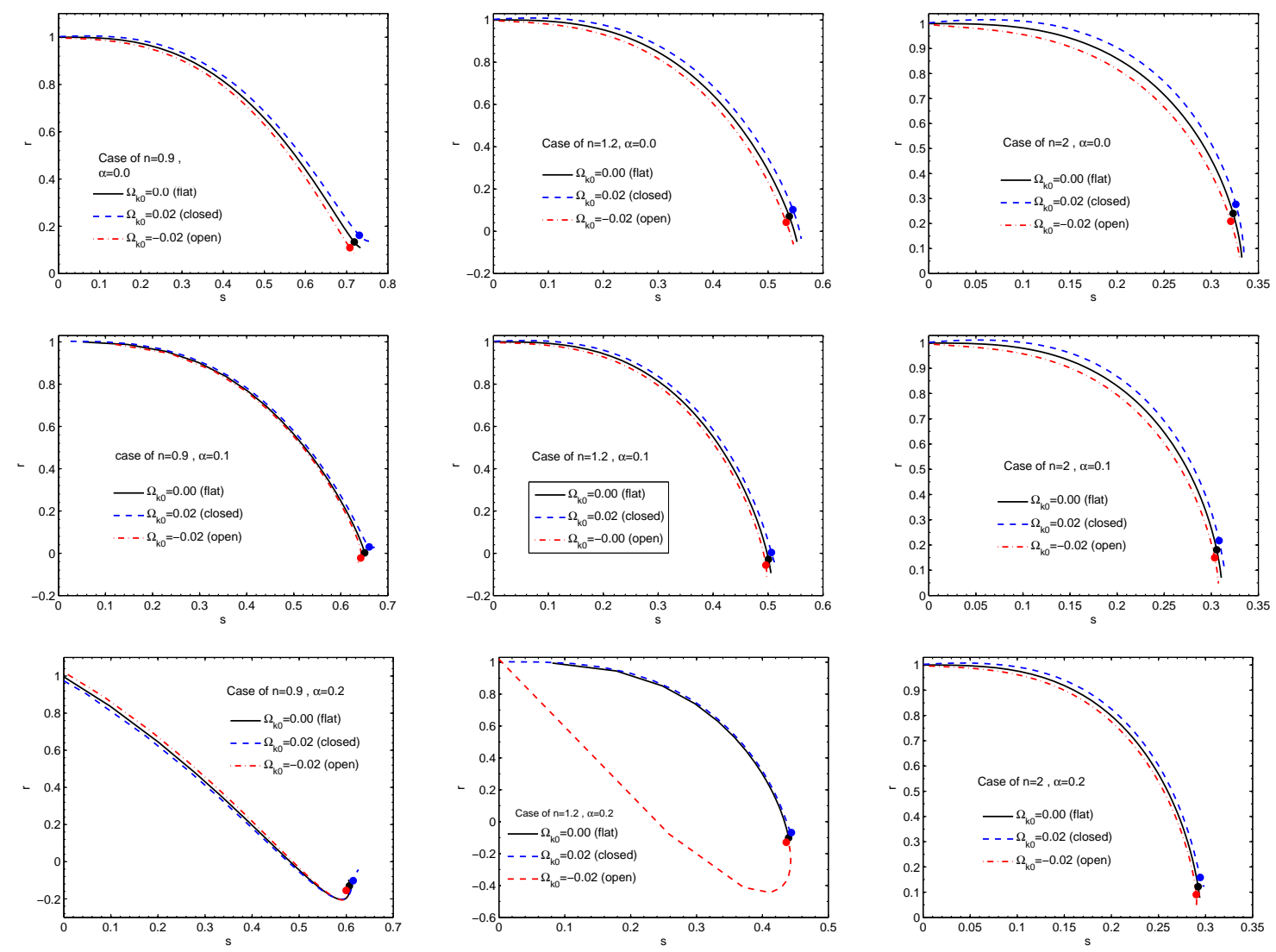

FIG. 3: The evolutionary trajectories of the statrefinder in the $r-s$ plane for different model parameters $n$ and $\alpha$ as well as different contribution of spatial curvatures. In the first arrow panels the ADE model is considered without the interaction between dark matter and dark energy, while in the second and third arrows the interaction term is taking into account. The statefinder parameters for the $\Lambda \mathrm{CAM}$ model corresponds to the fixed point $\{\mathrm{r}=1, \mathrm{~s}=0\}$ in the $r-s$ plane. 

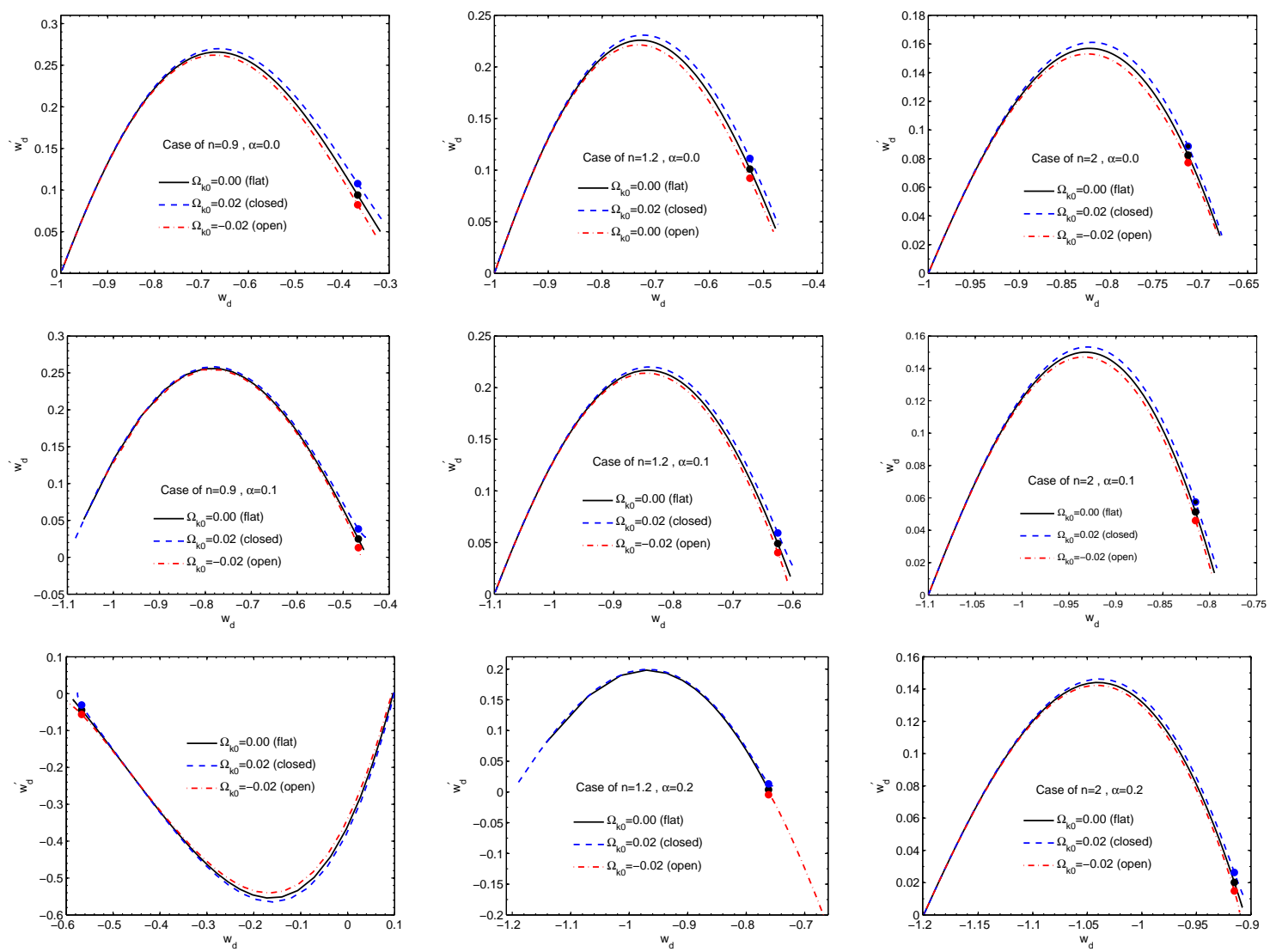

FIG. 4: The evolutionary trajectories of $\left\{w_{d}, w_{d}^{\prime}\right\}$ for different model parameters $n$ and $\alpha$ as well as different contribution of spatial curvatures. In the first arrow panels the ADE model is considered without the interaction between dark matter and dark energy, while in the second and third arrows the interaction term is taking into account. The $\left\{w, w^{\prime}\right\}$ for the $\Lambda$ CAM model corresponds to the fixed point $\left\{w=-1, w^{\prime}=0\right\}$ in the $w-w^{\prime}$ plane. 